\title{
Chronotherapy with a Renin-angiotensin System Inhibitor Ameliorates Renal Damage by Suppressing Intrarenal Renin-angiotensin System Activation
}

\author{
Taro Aoki ${ }^{1}$, Naro Ohashi ${ }^{1}$, Shinsuke Isobe ${ }^{1}$, Sayaka Ishigaki ${ }^{2}$, Takashi Matsuyama ${ }^{1}$, \\ Taichi Sato ${ }^{1}$, Tomoyuki Fujikura ${ }^{1}$, Akihiko Kato ${ }^{2}$, Hiroaki Miyajima ${ }^{1}$ and Hideo Yasuda ${ }^{1}$
}

\begin{abstract}
:
Objective The intrarenal renin-angiotensin system (RAS) is activated in chronic kidney disease (CKD) patients and is not suppressed at night in CKD patients showing nocturnal hypertension, contributing to renal damage. Furthermore, changes in RAS inhibitor administration from morning to evening, namely chronotherapy, ameliorates renal damage at night. We attempted to clarify whether or not chronotherapy ameliorates renal damage by suppressing the intrarenal RAS activity.

Methods We recruited 34 CKD patients with RAS inhibitors in the morning. We conducted ambulatory blood pressure (BP) monitoring and urine collection and evaluated urinary albumin (Alb) and angiotensinogen (AGT), which are surrogate markers for intrarenal RAS activity during the day and at night, respectively. The same experiments were conducted after changing the administration time. The ratio of values associated with morning versus evening dosing was defined as the morning to evening (M/E) ratio.

Results The M/E ratio of urinary Alb had a significant and positive relationship with that of urinary AGT during the day and at night in all CKD patients. However, no significant relationships were found between the M/E ratios of urinary Alb and AGT using multiple linear regression analyses. Conversely, there was a significant and positive relationship between the M/E ratios of urinary Alb and AGT at night but not during the day in CKD patients whose estimated glomerular filtration rate was $<45 \mathrm{~mL} / \mathrm{min} / 1.73 \mathrm{~m}^{2}$ and whose night-today ratio of systolic BP was $>0.90$, even after adjustment.

Conclusion This study indicated that chronotherapy with RAS inhibitors improved the renal damage via intrarenal RAS suppression, especially in CKD patients with an impaired renal function and nocturnal hypertension.
\end{abstract}

Key words: chronic kidney disease, chronotherapy, intrarenal renin-angiotensin system, renin-angiotensin system inhibitor, urinary angiotensinogen

(Intern Med 59: 2237-2244, 2020)

(DOI: 10.2169/internalmedicine.4243-19)

\section{Introduction}

Hypertension as well as the disruption of the diurnal blood pressure (BP) variations is a major risk factor for cardiovascular disease (CVD), stroke, and end-stage renal disease (1-4). Circadian BP rhythms are classified as extreme dipper, dipper, non-dipper, or riser patterns when the night- to-day (N/D) ratio of systolic BP is $<0.80,0.80$ to $<0.90$, 0.90-1.00, and $>1.00$, respectively (5). The BP circadian rhythm of normal subjects generally shows a dipper pattern. Conversely, chronic kidney disease (CKD) patients frequently show non-dipper and riser patterns, which are associated with a poor prognosis for CVD and stroke $(5,6)$.

The circulating renin-angiotensin system (RAS) is known to play a critical role in the regulation of arterial pressure

${ }^{1}$ Internal Medicine 1, Hamamatsu University School of Medicine, Japan and ${ }^{2}$ Blood Purification Unit, Hamamatsu University School of Medicine, Japan

Received for publication November 22, 2019; Accepted for publication February 24, 2020

Correspondence to Dr. Naro Ohashi, ohashi-n@hama-med.ac.jp 
and sodium homeostasis (7). Furthermore, it has been shown that intrarenal RAS is activated in some animal models and patients with CKD or hypertension and that this activation plays a critical role in the pathophysiology of renal damage, independent of circulating RAS (8-12).

We investigated the circadian rhythm of urinary angiotensinogen (U-AGT), which reflects intrarenal RAS activity in CKD patients classified by circadian BP rhythms, and clarified that U-AGT levels did not decrease at night versus during the day in CKD patients, showing a riser pattern of circadian BP. In addition, we showed that circadian fluctuations of albuminuria and proteinuria occurred parallel to that of U-AGT levels, that U-AGT levels were significantly and positively correlated with BP levels, and that circadian fluctuations of U-AGT were correlated with diurnal BP changes (8).

The change in administration time of antihypertensive drugs from morning to bedtime, namely chronotherapy, has recently been recommended to decrease the BP throughout the day, especially at night (13-15), and ameliorate CVD $(16,17)$ and renal damage (18). However, whether or not chronotherapy alters the intrarenal RAS activity and whether or not these changes reflect the changes in renal damage has not been clarified.

\section{Materials and Methods}

\section{Patients}

This study was approved by the ethics committee of Hamamatsu University School of Medicine and adhered to the principles of the Declaration of Helsinki. We recruited 34 CKD patients who took RAS inhibitors: 31 took an angiotensin II (Ang II) receptor blocker (ARB) (valsartan in 4, candesartan in 5 , losartan in 6 , telmisartan in 6 , azilsartan in 3 , irbesartan in 4 , and olmesartan in 3), while 3 took an angiotensin-converting enzyme inhibitor (ACE-I) (temocapril in 1, enalapril in 2) in the morning and were admitted to our hospital between March 2013 and January 2018.

Written informed consent was obtained from all patients. We excluded those patients undergoing hemodialysis or peritoneal dialysis.

\section{Study protocols}

We started the experiment the day after admission while continuing the RAS inhibitor dosage in the morning (8:00 am). We collected urine during the day (6:00 AM to 9:00 $\mathrm{PM})$ and at night (9:00 PM to 6:00 AM). Ambulatory BP monitoring (ABPM) was conducted at 30-minute intervals during the day and at night using an automatic device (TM2431; A and D, Tokyo, Japan). We divided the 24-hour ABPM into day and night segments using the sleep and waking times recorded in the patients' behavior records. Blood samples were also drawn at 9:00 PM and 6:00 AM the next day after the patients had rested in the supine position for at least 15 minutes. The blood samples drawn at
9:00 PM and 6:00 AM were considered the samples obtained at the end of the day and night periods, as described previously $(8,19-21)$.

Blood samples were centrifuged at 3,000 rpm for $10 \mathrm{~min}-$ utes at $4^{\circ} \mathrm{C}$, and urinary samples were centrifuged at 1,500 rpm for 5 minutes at $4{ }^{\circ} \mathrm{C}$. Both samples were stored at $-80^{\circ} \mathrm{C}$ until the assays were conducted. The same experiments were conducted a mean $4.1 \pm 0.48$ days after changing the RAS inhibitor administration timing to the evening (7:00 PM). The patients took $2.3 \pm 1.4$ antihypertension medications upon admission, and their physicians were not permitted to increase or decrease the number or dose of antihypertensive drugs during the experiments. Patients consumed a hospitalserved diet containing $8 \mathrm{~g}$ salt/day (standard salt diet) (4 patients) or $6 \mathrm{~g}$ salt/day (low salt diet) (30 patients). Each patient's diet was determined by their physicians.

\section{Clinical data}

The patients' clinical data, such as their age, sex, and body mass index (BMI), were recorded at the time of admission. During the 24-hour ABPM, the BP was measured noninvasively every 30 minutes as described above. During the day, BP values were calculated as the mean of the readings during waking hours, whereas at night, BP values were the mean of the remaining values. Circadian BP rhythms were classified according to the N/D ratio of systolic BP, as described previously (5). Serum and urinary creatinine concentrations were measured in the clinical laboratory of the Hamamatsu University School of Medicine, University Hospital. The levels of U-AGT, which is known to be a surrogate marker of intrarenal RAS activity $(11,12)$, were measured using an enzyme-linked immunosorbent assay, as described previously (22). Urinary albumin (U-Alb) concentrations were determined using a radioimmunoassay (SRL, Tokyo, Japan). Serum creatinine concentrations were measured in blood drawn at 9:00 PM and 6:00 AM, and the estimated glomerular filtration rate (eGFR) was calculated using the serum creatinine concentrations in the Japanese eGFR equation (23). The daytime and nighttime excretion ratios of $\mathrm{U}$ Alb/creatinine (U-Alb/Cr) and U-AGT/creatinine (U-AGT/ $\mathrm{Cr}$ ) were calculated. Daily urinary sodium excretion was collected, and the daily salt intake levels were determined by dividing the daily urinary sodium excretion by 17 . In addition, daily urinary urea nitrogen was collected, and the daily protein intake level was calculated using the MaroniMitch formula (24). The ratios of the clinical parameters associated with morning versus evening dosage were defined as the morning to evening $(\mathrm{M} / \mathrm{E})$ ratios.

\section{Statistical analyses}

The results are expressed as the mean \pm standard deviation. The significance of differences between dosing in the morning versus evening was determined using Student's $t$ test for paired samples. Because the U-Alb/Cr and U-AGT/ $\mathrm{Cr}$ values were not normally distributed, logarithmic transformation was applied to them. Thereafter, Student's $t$-test 
Table 1. Patient Characteristics.

\begin{tabular}{|c|c|}
\hline Age (year) & $60.2 \pm 19.4$ \\
\hline Sex & Male 22 / Female 12 \\
\hline Height $(\mathrm{cm})$ & $163.3 \pm 9.4$ \\
\hline Body weight $(\mathrm{kg})$ & $62.9 \pm 10.2$ \\
\hline Body mass index $\left(\mathrm{kg} / \mathrm{m}^{2}\right)$ & $23.6 \pm 3.6$ \\
\hline Causes of CKD & $\begin{array}{c}\text { DKD: } 7 \text { / CGN: } 21 \text { / NS: } 5 \text { / } \\
\text { Other } 1\end{array}$ \\
\hline Comorbidity & $\begin{array}{l}\text { DM: Present: } 9 \text { / Absent: } 25 \\
\text { HT: Present: } 32 \text { / Absent: } 2\end{array}$ \\
\hline $\mathrm{eGFR}\left(\mathrm{mL} / \mathrm{min} / 1.73 \mathrm{~m}^{2}\right)$ & $34.8 \pm 30.8$ \\
\hline CKD stage & $\begin{array}{l}\text { Stage G1: } 2 \text { / Stage G2: } 5 \text { / } \\
\text { Stage G3a: 7/ Stage G3b: } 2 \text { / } \\
\text { Stage G4: } 3 \text { / Stage G5: } 15\end{array}$ \\
\hline Circadian BP rhythm & $\begin{array}{l}\text { Extreme dipper: } 0 \text { / Dipper: } 7 \text { / } \\
\text { Non-dipper: } 15 \text { / Riser: } 10\end{array}$ \\
\hline \multicolumn{2}{|l|}{ Hypertension treatment } \\
\hline Number of medications & $2.3 \pm 1.4$ \\
\hline 1 medication $(\%)$ & 32.4 \\
\hline 2 medications (\%) & 38.2 \\
\hline$\geq 3$ medications $(\%)$ & 29.5 \\
\hline $\mathrm{ARB}(\%)$ & 91.2 \\
\hline ACE-I (\%) & 8.8 \\
\hline $\mathrm{CCB}(\%)$ & 61.8 \\
\hline$\alpha$-blocker $(\%)$ & 14.7 \\
\hline$\beta$-blocker (\%) & 5.9 \\
\hline Diuretic (\%) & 23.5 \\
\hline
\end{tabular}

CKD: chronic kidney disease, DKD: diabetic kidney disease, CGN: chronic glomerulonephritis, NS: nephrosclerosis, DM: diabetes mellitus, HT: hypertension, eGFR: estimated glomerular filtration rate, BP: blood pressure, ARB: angiotensin II receptor blocker, ACE-I: angiotensin converting enzyme inhibitor, CCB: calcium channel blocker

was performed. The correlations between the $\mathrm{M} / \mathrm{E}$ ratio of $\mathrm{U}-\mathrm{Alb} / \mathrm{Cr}$ and $\mathrm{M} / \mathrm{E}$ ratios of $\mathrm{U}-\mathrm{AGT} / \mathrm{Cr}$, systolic BP, or other clinical parameters were evaluated using Pearson's productmoment correlation test. Multiple linear regression analyses were conducted to evaluate the relationships between the M/ E ratio of $\mathrm{U}-\mathrm{Alb} / \mathrm{Cr}$ and the $\mathrm{M} / \mathrm{E}$ ratio of $\mathrm{U}-\mathrm{AGT} / \mathrm{Cr}$. The age, sex, BMI, eGFR, and systolic BP were selected as independent variables since they are commonly included in multiple linear regression analyses. We considered values of $\mathrm{p}<0.05$ as statistically significant. Statistical analyses were performed using IBM $^{\circledR}$ SPSS $^{\circledR}$ software version 25 (IBM Corporation, Armonk, USA).

\section{Results}

\section{Patient characteristics}

Thirty-four CKD patients who took RAS inhibitors in the morning and were admitted to our hospital during the study duration were included in this study. Their baseline characteristics are presented in Table 1. Most patients were of middle to advanced age $(60.2 \pm 19.4$ years). The patients' renal function had deteriorated moderately to severely (eGFR, $34.8 \pm 30.8 \mathrm{~mL} / \mathrm{min} / 1.73 \mathrm{~m}^{2}$ ). Twenty-four-hour ABPM could not be performed in two patients.

\section{The comparison of each parameter with morning versus evening dosage of RAS inhibitors in all pa- tients}

Table 2 shows the changes in each parameter between morning and evening dosage of RAS inhibitors in all patients. The BP levels and U-Alb and U-AGT excretion were significantly decreased during the day and at night by chronotherapy. In contrast, the intake of both salt and protein was similar regardless of dosage timing.

Relationships between the M/E ratios of U-Alb excretion and clinical parameters during the day and at night

We investigated the correlations between the M/E ratios of the day and nighttime U-Alb excretion and clinical parameters, such as the U-AGT excretion, and systolic and diastolic BP (Table 3). We used the $\mathrm{M} / \mathrm{E}$ ratio to evaluate the effectiveness of chronotherapy. The M/E ratio of the UAlb excretion was significantly and positively correlated with the M/E ratio of the day and nighttime U-AGT excretion (during the day, $\mathrm{r}=0.38, \mathrm{p}=0.041$; at night, $\mathrm{r}=0.55$, $\mathrm{p}<$ 0.01 ). Conversely, there were no significant relationships between the M/E ratios of the day and nighttime U-Alb excretion and those of BPs and heart rates.

Multiple linear regression analyses between the M/E ratios of U-Alb excretion and urinary AGT excretion during the day and at night

We performed multiple linear regression analyses to evaluate the relationships between the M/E ratios of $\mathrm{U}-\mathrm{Alb}$ and U-AGT excretion during the day and at night (Table 4). No significant relationship was found between the M/E ratio of U-Alb and urinary AGT excretion during the day or at night after adjusting for the age, sex, BMI, eGFR, and systolic $\mathrm{BP}$, as the multiple regression equation was not accepted during the day $(\mathrm{r}=0.59, \mathrm{p}=0.18)$ or at night $(\mathrm{r}=0.64$, $\mathrm{p}=0.11$ ).

The comparison of parameters concerning morning vs. evening dosage in 20 CKD patients with an eGFR $<45 \mathrm{~mL} / \mathrm{min} / 1.73 \mathrm{~m}^{2}$

The patients in this study were a heterogeneous population with a varied renal function. Therefore, we performed subgroup analyses in the 20 patients with moderate to severe renal impairment (eGFR $<45 \mathrm{~mL} / \mathrm{min} / 1.73 \mathrm{~m}^{2}$ ). Supplementary material 1 shows the changes in each parameter before and after chronotherapy of RAS inhibitors during the day, at night, and all day. The BP and U-Alb excretion were significantly decreased by chronotherapy in this subgroup during the day and at night. There was also a significant decrease in the U-AGT excretion at night. However, the U-AGT excretion during the day tended to be decreased by chronotherapy. Therefore, we conducted additional analyses focusing on the nighttime period as the main therapeutic target of 
Table 2. Comparison of Each Parameter in Morning Versus Evening Dosage of Renin-angiotensin System Inhibitors in All Patients.

\begin{tabular}{lccc}
\hline & Morning dosage & Evening dosage & p value \\
\hline N/D ratio of SBP & $0.96 \pm 0.066$ & $0.94 \pm 0.072$ & 0.19 \\
SBP (mmHg) (all day) & $129.8 \pm 18.1$ & $124.3 \pm 15.6$ & $<0.01$ \\
DBP (mmHg) (all day) & $74.7 \pm 9.9$ & $71.9 \pm 8.4$ & $<0.01$ \\
Heart rate (/min) (all day) & $68.5 \pm 5.4$ & $67.8 \pm 5.7$ & 0.41 \\
SBP (mmHg) (daytime) & $131.4 \pm 18.4$ & $126.4 \pm 15.9$ & $<0.01$ \\
DBP (mmHg) (daytime) & $76.3 \pm 9.7$ & $73.5 \pm 8.9$ & $<0.01$ \\
Heart rate $(/ \mathrm{min}$ ) (daytime) & $70.3 \pm 6.2$ & $69.6 \pm 7.0$ & 0.45 \\
SBP (mmHg) (nighttime) & $125.1 \pm 18.5$ & $118.4 \pm 16.0$ & $<0.01$ \\
DBP (mmHg) (nighttime) & $72.5 \pm 9.5$ & $68.2 \pm 9.0$ & $<0.01$ \\
Heart rate $(/ \mathrm{min})$ (nighttime) & $63.2 \pm 8.5$ & $62.8 \pm 7.3$ & 0.60 \\
Log U-Alb/Cr (mg/gCr) (daytime) & $3.02 \pm 0.49$ & $2.94 \pm 0.47$ & 0.018 \\
Log U-AGT/Cr ( $\mu$ g/gCr) (daytime) & $2.51 \pm 0.82$ & $2.42 \pm 0.82$ & 0.044 \\
Log U-Alb/Cr (mg/gCr) (nighttime) & $2.88 \pm 0.55$ & $2.82 \pm 0.52$ & 0.031 \\
Log U-AGT/Cr ( $\mu$ g/gCr) (nighttime) & $2.37 \pm 0.90$ & $2.27 \pm 0.88$ & 0.021 \\
Salt intake (g/day) & $5.92 \pm 2.30$ & $5.56 \pm 2.80$ & 0.55 \\
Protein intake (g/day) & $51.0 \pm 15.1$ & $52.6 \pm 23.7$ & 0.62 \\
\hline
\end{tabular}

N/D ratio: night-to-day ratio, SBP: systolic blood pressure, DBP: diastolic blood pressure, Cr: creatinine, U-Alb: urinary albumin, U-AGT: urinary angiotensinogen

Table 3. Relationships between Morning-to-evening (M/E) Ratios of Urinary Albumin Excretion Levels and Clinical Parameters during Daytime and Nighttime.

\begin{tabular}{lccccc}
\hline & \multicolumn{2}{c}{ Daytime } & \multicolumn{2}{c}{ Nighttime } \\
& $\mathrm{r}$ & $\mathrm{p}$ & $\mathrm{r}$ & $\mathrm{p}$ \\
\hline M/E ratio of Log U-AGT/Cr (daytime) & 0.38 & 0.041 & & \\
M/E ratio of Log U-AGT/Cr (nighttime) & & & 0.55 & $<0.01$ \\
M/E ratio of SBP (daytime) & 0.36 & 0.054 & & \\
M/E ratio of SBP (nighttime) & & & 0.27 & 0.18 \\
M/E ratio of DBP (daytime) & 0.25 & 0.19 & & \\
M/E ratio of DBP (nighttime) & & & -0.024 & 0.91 \\
M/E ratio of heart rate (daytime) & -0.090 & 0.65 & & \\
M/E ratio of heart rate (nighttime) & & & 0.18 & 0.36 \\
\hline U-AGT: urinary angiotensinogen, Cr: creatinine, SBP: systolic blood pressure, DBP: dia-
\end{tabular}
stolic blood pressure

chronotherapy.

Relationships between the M/E ratio of the U-Alb excretion vs. clinical parameters, including the nighttime U-AGT excretion, in 20 CKD patients with an eGFR $<45 \mathrm{~mL} / \mathrm{min} / 1.73 \mathrm{~m}^{2}$

The M/E ratio of U-Alb excretion was significantly positively correlated with the $\mathrm{M} / \mathrm{E}$ ratios of the nighttime UAGT excretion ( $\mathrm{r}=0.69, \mathrm{p}<0.01)$ (Fig. 1) and nighttime systolic BP $(\mathrm{r}=0.48, \mathrm{p}=0.049)$ in $20 \mathrm{CKD}$ patients with an eGFR $<45 \mathrm{~mL} / \mathrm{min} / 1.73 \mathrm{~m}^{2}$. In contrast, there were no significant relationships between the $\mathrm{M} / \mathrm{E}$ ratio of the nighttime U-Alb excretion and that of diastolic BP $(r=-0.034, p=0.90)$ and heart rate $(\mathrm{r}=0.34, \mathrm{p}=0.16)$.
Multiple linear regression analyses between the M/E ratio of the nighttime $U$-Alb excretion and nighttime $U$-AGT excretion in 20 CKD patients with an eGFR < $45 \mathrm{~mL} / \mathrm{min} / 1.73 \mathrm{~m}^{2}$

We performed multiple linear regression analyses to evaluate the relationships between the $\mathrm{M} / \mathrm{E}$ ratios of the nighttime U-Alb excretion and the nighttime U-AGT excretion (Table 5). A significant positive relationship between the $\mathrm{M} / \mathrm{E}$ ratio of the nighttime U-Alb excretion and the nighttime U-AGT excretion was maintained even after adjusting for the age, sex, BMI, eGFR, and M/E ratio of systolic BP $(\beta=0.73, \mathrm{p}<0.01)$. 
Table 4. Multiple Linear Regression Analyses between Morning-toevening (M/E) Ratio of Urinary Albumin Excretion Levels and Urinary Angiotensinogen (U-AGT) Excretion Levels during Daytime and Nighttime.

\begin{tabular}{|c|c|c|c|c|}
\hline & \multicolumn{2}{|c|}{$\begin{array}{c}\text { Daytime } \\
\mathrm{r}=0.59, \mathrm{p}=0.18\end{array}$} & \multicolumn{2}{|c|}{$\begin{array}{c}\text { Nighttime } \\
\mathrm{r}=0.64, \mathrm{p}=0.11\end{array}$} \\
\hline & $\beta$ & $\mathrm{p}$ & $\beta$ & $\mathrm{p}$ \\
\hline Age (year) & 0.34 & 0.23 & 0.22 & 0.51 \\
\hline Sex & 0.27 & 0.21 & -0.15 & 0.45 \\
\hline Body mass index $\left(\mathrm{kg} / \mathrm{m}^{2}\right)$ & 0.23 & 0.27 & 0.18 & 0.36 \\
\hline eGFR $\left(\mathrm{mL} / \mathrm{min} / 1.73 \mathrm{~m}^{2}\right)$ (daytime) & 0.013 & 0.96 & & \\
\hline eGFR (mL/min/1.73m²) (nighttime) & & & 0.060 & 0.85 \\
\hline $\mathrm{M} / \mathrm{E}$ ratio of SBP (daytime) & 0.012 & 0.96 & & \\
\hline $\mathrm{M} / \mathrm{E}$ ratio of SBP (nighttime) & & & 0.17 & 0.40 \\
\hline M/E ratio of Log U-AGT/Cr (daytime) & 0.51 & 0.058 & & \\
\hline $\begin{array}{l}\mathrm{M} / \mathrm{E} \text { ratio of } \mathrm{Log} \mathrm{U}-\mathrm{AGT} / \mathrm{Cr} \text { (night- } \\
\text { time) }\end{array}$ & & & 0.51 & 0.016 \\
\hline
\end{tabular}

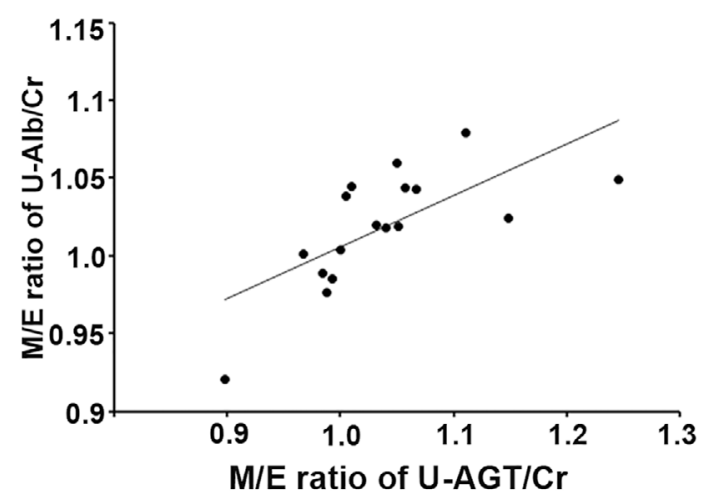

Figure 1. Relationships between the morning-to-evening $(\mathrm{M} / \mathrm{E})$ ratio of the urinary albumin (U-Alb) and urinary angiotensinogen (U-AGT) excretion at night in 20 chronic kidney disease patients with an estimated glomerular filtration rate $<45 \mathrm{~mL} / \mathrm{min} / 1.73 \mathrm{~m}^{2}$. Cr: creatinine

The comparison of each parameter for morning versus evening dosage in 25 CKD patients with nondipper and riser BP patterns

Next, we performed subgroup analyses for 25 CKD patients with nocturnal hypertension, namely those with nondipper and riser BP patterns. The relationship between the subgroup of $20 \mathrm{CKD}$ patients with an eGFR $<45 \mathrm{~mL} / \mathrm{min} /$ $1.73 \mathrm{~m}^{2}$ and the subgroup of $25 \mathrm{CKD}$ patients with nondipper and riser BP patterns is shown in Supplementary material 2. More than half of the cases overlapped between the two groups, as the proportions of non-dipper and riser patients increased with the decreasing renal function (25). In contrast, there were 10 patients who showed nocturnal hypertension despite relatively mild renal impairment.

The N/D ratio of SBP was significantly decreased by chronotherapy (morning dosage, $0.98 \pm 0.052$; evening dos-
Table 5. Multiple Linear Regression Analyses between Morning-to-evening (M/E) Ratio of Nighttime Urinary Albumin Excretion Levels and Nighttime Urinary Angiotensinogen (U-AGT) Excretion Levels in 20 Chronic Kidney Disease Patients with an Estimated Glomerular Filtration Rate (eGFR) Less than $45 \mathrm{~mL} / \mathrm{min} / 1.73 \mathrm{~m}^{2}$.

\begin{tabular}{lcc}
\hline & \multicolumn{2}{c}{ Nighttime } \\
& $\mathrm{r}=0.87, \mathrm{p}=0.039$ \\
\hline Age (year) & -0.15 & 0.45 \\
Sex & 0.16 & 0.43 \\
Body mass index $\left(\mathrm{kg} / \mathrm{m}^{2}\right)$ & 0.041 & 0.84 \\
eGFR (mL/min/1.73m ${ }^{2}$ (nighttime) & 0.11 & 0.60 \\
M/E ratio of SBP $($ nighttime) & 0.31 & 0.15 \\
M/E ratio of Log U-AGT/Cr (nighttime) & 0.73 & $<0.01$ \\
\hline SBP: systolic blood pressure, Cr: creatinine & &
\end{tabular}

age, $0.96 \pm 0.068 ; \mathrm{p}=0.035)$. In addition, similar to the findings in the patients with moderate to severe renal impairment, the daytime and nighttime $\mathrm{BP}$ and $\mathrm{U}-\mathrm{Alb}$ excretion and the nighttime U-AGT excretion were significantly decreased by chronotherapy (Supplementary material 3).

Relationships between M/E ratio of U-Alb excretion and clinical parameters including U-AGT excretion at night in 25 CKD patients with non-dipper and riser BP patterns

Fig. 2 shows the correlations between the M/E ratio of the nighttime U-Alb and U-AGT excretion $(r=0.80, \mathrm{p}<0.01)$. No significant relationships were found between the M/E ratio of U-Alb excretion and that of the systolic BP ( $\mathrm{r}=0.18$, $\mathrm{p}=0.43)$, diastolic $\mathrm{BP}(\mathrm{r}=0.21, \mathrm{p}=0.40)$, or heart rate $(\mathrm{r}=0.19$, $\mathrm{p}=0.41$ ) at night. 


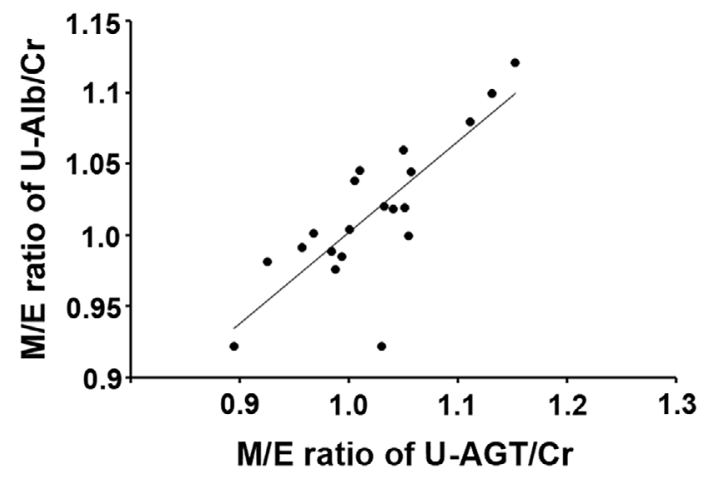

Figure 2. Relationships between the morning-to-evening $(\mathrm{M} / \mathrm{E})$ ratio of the urinary albumin (U-Alb) and urinary angiotensinogen (U-AGT) excretion at night in 25 chronic kidney disease (CKD) patients with non-dipper and riser blood pressure patterns. $\mathrm{Cr}$ : creatinine

Multiple linear regression analyses between M/E ratio of U-Alb excretion and U-AGT excretion at night in 25 CKD patients with non-dipper and riser BP patterns

We performed multiple linear regression analyses to evaluate the relationships between the $\mathrm{M} / \mathrm{E}$ ratio of the $\mathrm{U}$ Alb excretion and U-AGT excretion in 25 CKD patients with nocturnal hypertension. A significant positive relationship was found between the M/E ratios of the U-Alb excretion and U-AGT excretion at night, even after adjusting for the age, sex, BMI, eGFR, and M/E ratio of systolic BP ( $\beta=$ 1.04, $\mathrm{p}<0.01$ ) (Table 6).

\section{Discussion}

This study indicated that chronotherapy with RAS inhibitors reduced the U-Alb excretion during the day and at night and that there was a significant and positive relationship between the M/E ratios of the U-Alb excretion and U-AGT excretion at night, even after adjusting for the age, sex, BMI, eGFR, and systolic BP, especially in CKD patients with an impaired renal function and nocturnal hypertension.

Hermida et al. were the first to report a change in the administration time of one antihypertensive drug from morning to bedtime, namely chronotherapy, reduced the prevalence of non-dipping hypertension (26). Furthermore, it has been clarified that chronotherapy with several classes of antihypertensive drugs ameliorates organ damage, such as CVD and renal damage (16-18). Wang et al. also reported on chronotherapy with a RAS inhibitor. They performed a clinical study in 30 patients with a dipping BP pattern and 60 patients with a non-dipping BP pattern who were divided into 2 groups and treated with doses of valsartan (80-320 $\mathrm{mg}$ ) before bedtime or after waking up. Non-dipping BP patients treated with bedtime doses of valsartan showed a greater reduction in 24-hour and bedtime proteinuria and a greater delayed decline in the eGFR than patients with a
Table 6. Multiple Linear Regression Analyses between Morning-to-evening (M/E) Ratio of Urinary Albumin Excretion Levels and Urinary Angiotensinogen (U-AGT) Excretion Levels during Nighttime in $\mathbf{2 5}$ Chronic Kidney Disease (CKD) Patients with Non-dipper and Riser Blood Pressure Patterns.

\begin{tabular}{|c|c|c|}
\hline & \multicolumn{2}{|c|}{$\begin{array}{c}\text { Nighttime } \\
\mathrm{r}=0.86, \mathrm{p}<0.01\end{array}$} \\
\hline & $\beta$ & $\mathrm{p}$ \\
\hline Age (year) & -0.40 & 0.21 \\
\hline Sex & 0.017 & 0.92 \\
\hline Body mass index $\left(\mathrm{kg} / \mathrm{m}^{2}\right)$ & 0.049 & 0.77 \\
\hline eGFR (mL/min/1.73m²) (nighttime) & -0.52 & 0.077 \\
\hline $\mathrm{M} / \mathrm{E}$ ratio of SBP (nighttime) & -0.17 & 0.34 \\
\hline $\mathrm{M} / \mathrm{E}$ ratio of Log U-AGT/Cr (nighttime) & 1.04 & $<0.01$ \\
\hline
\end{tabular}

non-dipping BP pattern taking the same dose after waking up $(\mathrm{p}<0.05)(27)$. The present study showed that chronotherapy with RAS inhibitors ameliorates the levels of BP as well as U-Alb excretion, especially at night, which is similar to the findings in previous reports. In addition, we clarified for the first time that the levels of U-AGT excretion at night were significantly decreased by chronotherapy with an RAS inhibitor. However, we were unable to not determine why intrarenal RAS activation was suppressed by chronotherapy with an RAS inhibitor at night because of the clinical observational nature of this study. In the near future, we will perform an animal study to clarify the effectiveness of chronotherapy.

Similar to the previous reports, chronotherapy with RAS inhibitors showed a significant reduction in the U-Alb excretion and BP not only at night but also during the day in our study. In addition, we also noted that U-AGT excretion both during the day and at night was significantly decreased by chronotherapy in all CKD patients. However, multiple regression analyses showed no significant correlations between the $\mathrm{M} / \mathrm{E}$ ratios of the daytime and nighttime $\mathrm{U}-\mathrm{Alb}$ and $\mathrm{U}$ AGT excretion in all CKD patients. We consider this to be because the renal function of the CKD patients in this study ranged from mild to severe renal impairment. Farmer et al. examined the ambulatory BP in 380 patients with essential hypertension and showed that the prevalence of a nondipping pattern in the diurnal BP rhythm increased as the plasma creatinine level increased, regardless of the number of antihypertensives taken by the patients (25). In addition, we have also clarified that urinary AGT levels did not decrease at night compared with those during the day in CKD patients showing a riser pattern of circadian BP (8). These results indicate that renal dysfunction causes nocturnal hypertension and that nocturnal hypertension is associated with intrarenal RAS activation at night. Accordingly, we also evaluated $20 \mathrm{CKD}$ patients whose eGFR was $<45 \mathrm{~mL} / \mathrm{min} /$ $1.73 \mathrm{~m}^{2}$ and $25 \mathrm{CKD}$ patients who had non-dipper and riser 
patterns of BP and showed that chronotherapy with RAS inhibitors improved the renal damage via intrarenal RAS suppression, even after adjusting for the age, sex, BMI, eGFR, and systolic BP, especially at night.

In subgroup analyses, although no significant changes were found in the daytime U-AGT excretion, the U-Alb excretion was significantly reduced. It is difficult to elucidate the underlying mechanisms in this clinical study, and undetermined factors other than intrarenal RAS activity, such as BP, sympathetic nerve activity, and physical activity, may influence the U-Alb excretion during the day.

In previous reports, chronotherapy of antihypertensive drugs was performed to compare the effects by administration timing (waking and bedtime dosages) $(13,14,16,17$, 26). However, we performed a chronotherapy study that compared the effects between morning and evening dosages (8:00 AM and 7:00 PM, respectively). It is difficult to compare the effectiveness of chronotherapy between evening (our study) and bedtime dosages (previous studies), as the study designs are often different. For example, many studies were performed to compare the administration timing of antihypertensive drugs in different patients, and only a few studies, including ours, have compared the effectiveness before and after a change in the administration timing of antihypertensive drugs in the same patients. In addition, the number of changes in antihypertensive drugs for chronotherapy, follow-up period, and recruited patients differ among studies.

However, the remarkable finding of our study is that the daytime BPs were significantly lower than those in previous studies. For example, Hermida et al. assigned 661 CKD patients to either take all prescribed antihypertensive drugs upon waking or at least one at bedtime. As a result, although the nighttime BP was significantly different between the waking and bedtime dosages (waking dosage: 122.6 \pm $21.3 \mathrm{mmHg}$; bedtime dosage: $116.7 \pm 16.8 \mathrm{mmHg}, \mathrm{p}<0.001$ ), the daytime systolic BP values were not significantly different between dosages (waking dosage: 128.3 $\pm 17.7 \mathrm{mmHg}$; bedtime dosage: $129.3 \pm 15.2 \mathrm{mmHg}, \mathrm{p}=0.419)$ (17). Based on these results it is presumed that an evening dosage decreased the daytime BP between 7:00 PM and bedtime more remarkably than a bedtime dosage. Further clinical studies to compare the effectiveness between nighttime and bedtime dosages are needed to clarify these findings.

Several limitations associated with the present study warrant mention. First, its sample size was relatively small, and patients were recruited from a single center. Nevertheless, we were able to determine that the $\mathrm{M} / \mathrm{E}$ ratio of $\mathrm{U}-\mathrm{Alb}$ excretion had a significant and positive relationship with the $\mathrm{M} / \mathrm{E}$ ratio of urinary AGT excretion at night after adjusting for the age, sex, BMI, eGFR, and systolic BP in only CKD patients with moderate to severe renal impairment and nocturnal hypertension. As the relatively small sample size may have contributed to a bias in the results, a larger study is needed in the future to confirm our results. Second, we recruited all CKD patients taking RAS inhibitors in the morn- ing, regardless of the type (ACE-Is or ARBs) or different drugs of ARBs. Because the mechanisms of ACE-Is or ARBs, antagonistic function and selectivity to Ang II type 1 receptor, and half-lives of each ARB differ, the antihypertensive effects and organ protective effects of these drugs also differ $(28,29)$. Therefore, it would be best to unify the RAS inhibitors. However, it was difficult to do so because of the real clinical practice setting and limited sample size. Indeed, the few reports concerning chronotherapy available in the literature are limited to a single kind of RAS inhibitor. However, we were able to clarify that chronotherapy using RAS inhibitors ameliorated renal damage through intrarenal RAS suppression, even after adjusting for some clinical parameters in CKD patients. Finally, it is possible that a lowsalt diet affected the results. Salt loading elevates the BP due to an increase in body fluid that is proportional to the amount of sodium in the body, while the BP levels decrease according to the degree of salt restriction (30). Furthermore, salt restriction is known to reduce the urinary protein excretion and suppress the progression of renal damage $(31,32)$. Recent studies have also found that salt loading induces renal damage independently of the increase in body fluids $(33,34)$. It is also known that salt loading activates the intrarenal RAS and induces renal damage in some kidney disease models (7). Therefore, as a potential cause for why the BP and U-Alb and U-AGT excretion were ameliorated with the evening versus morning dosage, it is possible that salt loading influenced the elevation of the BP and U-Alb and U-AGT excretion with the morning dosage because of the next day after admission and that a longer period of a low salt diet in evening dosage $(4.1 \pm 0.48$ days $)$ influenced the amelioration of these parameters. However, no significant differences in the salt intake were found between the morning and evening dosages $(5.92 \pm 2.30$ g/day vs. 5.56 \pm $2.80 \mathrm{~g} /$ day, respectively, $\mathrm{p}=0.55)$. In addition, there were no significant differences in the protein intake between the morning and evening dosages $(51.0 \pm 15.1$ g/day vs. $52.6 \pm$ $23.7 \mathrm{~g} /$ day, respectively, $\mathrm{p}=0.62$ ). These data suggest that the diet before and after admission did not markedly influence the study results. To clarify the actual effect of diet and switching chronotherapy using RAS inhibitors from a morning to evening dosage, a future study with a longer period of hospital diet intake and a study involving switching from an evening to morning dosage will be required. However, because the hospitalization duration is limited in our hospital, we were unable to extend the study period for our clinical research.

In conclusion, we clarified that chronotherapy using RAS inhibitors reduced the U-Alb excretion by suppressing the intrarenal RAS in CKD patients, especially at night; this effect was more remarkable in patients with a highly impaired renal function and nocturnal hypertension than in others. We consider it very useful to be able to reduce the BP and ameliorate renal damage using the simple and cost-effective method of changing the RAS inhibitor administration timing. Further studies are needed to validate our findings. 
The authors state that they have no Conflict of Interest (COI).

\section{Financial Support}

This work was supported by HUSM Grant-in-Aid.

\section{References}

1. Bakris GL, Williams M, Dworkin L, et al. Preserving renal function in adults with hypertension and diabetes: a consensus approach. National Kidney Foundation Hypertension and Diabetes Executive Committees Working Group. Am J Kidney Dis 36: 646661, 2000.

2. Ohkubo T, Hozawa A, Yamaguchi J, et al. Prognositic significance of the nocturnal decline in blood pressure in individuals with and without high 24-h blood pressure: the Ohasama study. J Hypertnes 20: 2183-2189, 2002.

3. Kario K, Pickering TG, Matsuo T, et al. Stroke prognosis and abnormal nocturnal blood pressure falls in older hypertensives. Hypertension 38: 852-857, 2001.

4. Agarwal R, Andersen MJ. Prognostic importance of ambulatory blood pressure recordings in patients with chronic kidney disease. Kidney Int 69: 1175-1180, 2006.

5. Kario K, Shimada K. Risers and extreme-dippers of nocturnal blood pressure in hypertension: antihypertensive strategy for nocturnal blood pressure. Clin Exp Hypertens 26: 177-189, 2004.

6. Boggia J, Li Y, Thijs L, et al.; International Database on Ambulatory blood pressure monitoring in relation to Cardiovascular Outcomes (IDACO) investigators. Prognostic accuracy of day versus night ambulatory blood pressure: a cohort study. Lancet 370: 1219-1229, 2007.

7. Kobori H, Nangaku M, Navar LG, et al. The intrarenal reninangiotensin system: from physiology to the pathobiology of hypertension and kidney disease. Pharmacol Rev 59: 251-287, 2007.

8. Isobe S, Ohashi N, Fujikura T, et al. Disturbed circadian rhythm of the intrarenal renin-angiotensin system: relevant to nocturnal hypertension and renal damage. Clin Exp Nephrol 19: 231-239, 2015.

9. Ohashi N, Katsurada A, Miyata K, et al. Activation of reactive oxygen species and the renin-angiotensin system in IgA nephropathy model mice. Clin Exp Pharmacol Physiol 36: 509-515, 2009.

10. Isobe $\mathrm{S}$, Ohashi $\mathrm{N}$, Ishigaki $\mathrm{S}$, et al. Augmented circadian rhythm of the intrarenal renin-angiotensin systems in anti-thymocyte serum nephritis rats. Hypertens Res 39: 312-320, 2016.

11. Kobori H, Alper AB Jr, Shenava R, et al. Urinary angiotensinogen as a novel biomarker of the intrarenal renin-angiotensin system status in hypertensive patients. Hypertension 53: 344-350, 2009.

12. Kobori H, Ohashi N, Katsurada A, et al. Urinary angiotensinogen as a potential biomarker of severity of chronic kidney diseases. J Am Soc Hypertens 2: 349-354, 2008.

13. Hermida RC, Ayala DE, Fernández JR, et al. Chronotherapy improves blood pressure control and reverts the nondipper pattern in patients with resistant hypertension. Hypertension 51: 69-76, 2008.

14. Almirall J, Comas L, Martínez-Ocaña JC, et al. Effects of chronotherapy on blood pressure control in non-dipper patients with refractory hypertension. Nephrol Dial Transplant 27: 1855-1859, 2012.

15. Hermida RC, Ayala DE, Mojón A, et al. Influence of time of day of blood pressure-lowering treatment on cardiovascular risk in hypertensive patients with type 2 diabetes. Diabetes Care 34: 12701276, 2011.

16. Hermida RC, Ayala DE, Mojón A, et al. Influence of circadian time of hypertension treatment on cardiovascular risk: results of the MAPEC study. Chronobiol Int 27: 1629-1651, 2010.

17. Hermida RC, Ayala DE, Mojón A, et al. Bedtime dosing of antihypertensive medications reduces cardiovascular risk in CKD. J Am Soc Nephrol 22: 2313-2321, 2011.
18. Minutolo R, Gabbai FB, Borrelli S, et al. Changing the timing of antihypertensive therapy to reduce nocturnal blood pressure in CKD: an 8-week uncontrolled trial. Am J Kidney Dis 50: 908917, 2007.

19. Ishigaki $\mathrm{S}$, Ohashi $\mathrm{N}$, Isobe $\mathrm{S}$, et al. Impaired endogenous nighttime melatonin secretion relates to intrarenal renin-angiotensin system activation and renal damage in patients with chronic kidney disease. Clin Exp Nephrol 20: 878-884, 2016.

20. Ohashi $\mathrm{N}$, Isobe $\mathrm{S}$, Ishigaki $\mathrm{S}$, et al. The effects of unilateral nephrectomy on blood pressure and its circadian rhythm. Intern Med 55: 3427-3433, 2016.

21. Fukuda M, Mizuno M, Yamanaka T, et al. Patients with renal dysfunction require a longer duration until blood pressure dips during the night. Hypertension 52: 1155-1160, 2008.

22. Katsurada A, Hagiwara Y, Miyashita K, et al. Novel sandwich ELISA for human angiotensinogen. Am J Physiol Renal Physiol 293: F956-F960, 2007.

23. Matsuo S, Imai E, Horio $M$, et al. Collaborators developing the Japanese equation for estimated GFR. Revised equations for estimated GFR from serum creatinine in Japan. Am J Kidney Dis 53: 982-992, 2009.

24. Maroni BJ, Steinman TI, Mitch WE. A method for estimating nitrogen intake of patients with chronic renal failure. Kidney Int 27: 58-65, 1985.

25. Farmer CK, Goldsmith DJ, Cox J, et al. An investigation of the effect of advancing uraemia, renal replacement therapy and renal transplantation on blood pressure diurnal valiability. Nephrol Dial Transplant 12: 2301-2307, 1997.

26. Hermida RC, Ayala DE, Calvo C, et al. Effects of time of day of treatment on ambulatory blood pressure pattern of patients with resistant hypertension. Hypertension 46: 1053-1059, 2005.

27. Wang C, Zhang J, Liu X, et al. Effect of valsartan with bedtime dosing on chronic kidney disease patients with nondipping blood pressure pattern. J Clin Hypertens 15: 48-54, 2013.

28. Miura S, Fujino M, Saku K. Angiotensin II receptor blocker as an inverse agonist: a current perspective. Curr Hypertens Rev 1: 115121, 2005.

29. Smith DH, Dubiel R, Jones M. Use of 24-hour ambulatory blood pressure monitoring to assess antihypertensive efficacy: a comparison of olmesartan medoxomil, losartan potassium, valsartan, and irbesartan. Am J Cardiovasc Drugs 5: 41-50, 2005.

30. Sacks FM, Svetkey LP, Vollmer WM, et al.; DASH-Sodium Collaborative Research Group. Effects on blood pressure of reduced dietary sodium and the Dietary Approaches to Stop Hypertension (DASH) diet. DASH-Sodium Collaborative Research Group. N Engl J Med 344: 3-10, 2001.

31. Swift PA, Markandu ND, Sagnella GA, et al. Modest salt reduction reduces blood pressure and urine protein excretion in black hypertensives: a randomized control trial. Hypertension 46: 308312, 2005.

32. Slagman MC, Waanders F, Hemmelder MH, et al.; HOlland NEphrology STudy Group. Moderate dietary sodium restriction added to angiotensin converting enzyme inhibition compared with dual blockade in lowering proteinuria and blood pressure: randomised controlled trial. BMJ 343: d4366, 2011.

33. Kim YH, Hwang JH, Noh JR, et al. Prevention of salt-induced renal injury by activation of $\mathrm{NAD}(\mathrm{P}) \mathrm{H}$ :quinone oxidoreductase 1 , associated with NADPH oxidase. Free Radic Biol Med 52: 880888, 2012.

34. Zhang M, Qin DN, Suo YP, et al. Endogenous hydrogen peroxide in the hypothalamic paraventricular nucleus regulates neurohormonal excitation in high salt-induced hypertension. Toxicol Lett 235 : 206-215, 2015.

The Internal Medicine is an Open Access journal distributed under the Creative Commons Attribution-NonCommercial-NoDerivatives 4.0 International License. To view the details of this license, please visit (https://creativecommons.org/licenses/ by-nc-nd/4.0/).

(C) 2020 The Japanese Society of Internal Medicine

Intern Med 59: 2237-2244, 2020 